\title{
2 PLATELET-RICH PLASMA: A 'FEELING' AND 'HOPE' AILING ATHLETES
}

Vijay D Shetty Consultant Orthopaedic Surgeon, Hiranandani Orthopaedic Medical Education (HOME), Dr LH Hiranandani Hospital Powai, Mumbai, India

\section{$10.1136 /$ bjsm.2010.078725.2}

Introduction A number of orthopaedic and sports conditions remain a major therapeutic challenge to orthopaedic surgeons even in this day and age of technological advance. Some of these difficult conditions include plantar fasciitis (chronic pain in the sole of the foot), tennis elbow (chronic pain in the elbow), ligament and muscle injuries (around the knee and other joints) and tendo-achelles (heel) injuries. Persistent symptoms, due to these conditions, in young sporty individuals have lead to premature ending of their competitive careers. Sports medicine experts all over the world, for a very long time, have been looking at the various options available to treat these conditions effectively. In this direction, platelet-rich plasma (PRP) therapy is taking the sports medicine world by storm, as is seen in recent years. PRP is now being effectively used to mend ligaments and repair tendon injuries, thanks to the recent technological surge.

Platelets Platelets are small disc or plate-like structures normally found in blood. They are tiny cells, yet powerful, that are partially responsible for blood clotting (stops bleeding), and are critical to the healing process. They promote more rapid healing by secreting healing and growth factors and thereby enhance recovery.

Platelet-rich plasma Platelet-rich plasma is the name given to plasma (normal component of blood) with a high concentration of platelets. When blood is taken from the patient and placed into a tube and spun in a centrifuge (a device that spins), it separates into several different components, one of which is called the platelet-rich plasma. Each cubic millimetre of this plasma solution can contain 1.5-2 million platelets. These concentrated platelets contain huge doses of very important bioactive proteins, such as growth factors, that are necessary for the repair and regeneration of tissues. These special proteins can also initiate new blood vessel formation, bone regeneration and healing, connective tissue repair and endorse overall wound healing.

Platelet-rich plasma therapy (biologic therapy) Whole blood (about $30 \mathrm{ml}$ ) is drawn from the patient, prior to the injection, in the blood bank. The blood is then centrifuged to separate the plasma (buffy coat) from the blood. This concentrate, which contains platelets and growth factors (multiplied several fold), is then mixed with activating agents and is injected back into the patient's own damaged tissue where it begins to initiate the process of repair. The repair response in the injured tissue is kick-started by the formation of a blood 
clot. This is then followed by the implanted platelets being dissolved, triggering the release of growth factors, which, in turn, leads to the formation of a fibrous scar tissue. Eventually, the injured tissue is completely replaced by healthy cells.

Safety of PRP The chances of an adverse reaction are completely nullified because the components used for treatment are derived from a person's own body. This makes the procedure entirely safe. Thus, there is no risk of transmission of disease.

A word of caution PRP therapy is not a quick fix. Regeneration of injured tendons and ligaments will take time. Physical therapy may be continued a week after the procedure. A patient who has undergone PRP therapy may initially suffer some soreness or discomfort, especially at the injection site. Local icepacks and analgesics will help to resolve these symptoms.

Benefits Early results with PRP treatment are very encouraging. Research indicates that the procedure will gain prominence as the first choice of treatment, for many of the conditions listed above, for reasons both medical and financial. Some of the highlights of PRP are as follows:

- non-operative treatment

- based on body's own healing potential

- can be considered when all doors are shut

- cheap compared with any other surgical procedures

- the entire procedure (admission, injection, recovery and discharge) lasts for not more than $4 \mathrm{~h}$

- it may reduce or even completely eliminate the need for complicated treatments like aggressive medications or surgery.

The future Although PRP therapy is gaining popularity in sports medicine, its use is fast spreading into other fields including dentistry, heart surgery and wound healing. It is exciting to learn that researchers are looking into its role in regenerating bone or helping arthritis. As both research and experience move forward, doctors see a wide range of clientele and applications on the horizon for PRP. A new international society, dedicated to research into the use of biological therapies in orthopaedics, was formed in the USA recently, of which the author is a founder member. 